\title{
Elimination of the internal electrostatic field in two- dimensional GaN-based semiconductors
}

\author{
Yuping Jia ${ }^{1}$, Zhiming Shi $\mathbb{D}^{1 凶}$, Wantong Hou ${ }^{1}$, Hang Zang ${ }^{1}$, Ke Jiang ${ }^{1}$, Yang Chen ${ }^{1}$, Shanli Zhang ${ }^{1}$, Zhanbin $\mathrm{Qi}^{1}$, Tong Wu ${ }^{1}$, \\ Xiaojuan Sun ${ }^{1}$ and Dabing Li (iD) ${ }^{1,2 凶}$
}

GaN-based semiconductors are promising materials for solid-state optoelectronic applications. However, the strong internal electrostatic field (IEF) along the [0001] direction is a serious problem that harms the efficiency of lighting devices based on GaNbased semiconductors due to the quantum confined Stark effect. Here we theoretically predict a method, reducing the dimensions from bulk to two-dimensional (2D) structures, to fundamentally remove the IEF. After thinning the materials to several nanometers, the wurtzite configuration (with strong IEF) spontaneously transform to the haeckelite (4|8) configuration (without IEF) due to the more stable neutral surface in the $4 \mid 8$ configuration. Meanwhile, the $4 \mid 8$ configuration maintain optoelectronic properties comparable to or even better than those of the wurtzite configuration. By carefully analyzing the interaction between $2 \mathrm{D}$ GaN and different types of substrates ( $\mathrm{SiC}$ and graphene), we not only provide clear physical insights for experimental results but also address a "thickness-controlled" vdW epitaxy scheme to experimentally realize the $4 \mid 8$ configuration. We believe that the $4 \mid 8$ configuration without IEF is a prospective material for diverse optoelectronic applications. In addition, we propose a point of view in engineering the properties of GaN-based semiconductors.

npj 2D Materials and Applications (2020)4:31; https://doi.org/10.1038/s41699-020-00165-1

\section{INTRODUCTION}

GaN-based semiconductors are highlighted for their excellent properties, such as tunable and direct band gap, high thermal conductivity, and good chemical stability ${ }^{1}$. The materials and devices have been extensively investigated in recent decades, which has led to significant progress in light-emitting diodes ${ }^{2-6}$, photodetectors $^{7-9}$, and laser diodes ${ }^{10-12}$. However, the great success in lighting devices is mainly limited by the polar material growth along the c-axis [0001]. GaN-based semiconductors crystallize into the wurtzite structure with a $c: a$ ratio lower than that for perfectly hexagonally close-packed atoms ( $c: a=1.633$ for perfect hcp, 1.601, 1.627, and 1.623 for AIN, GaN, and InN, respectively). This, together with the lack of inversion symmetry and the strong ionic nature of III- $\mathrm{N}$ bonds, produces a spontaneous dipole moment along the [0001] direction. The dipole moment causes opposite bound polarization charges on the metal-polar [0001] and N-polar [000-1] surfaces, which leads to a strong internal electrostatic field (IEF) along the [0001] direction ${ }^{13-15}$. The IEF separates electrons and holes spatially on either side and induces an energy band slope ${ }^{16}$, thus reducing the emission efficiency and leading to a redshift in lighting devices (quantum confined Stark effect) ${ }^{17,18}$. Therefore, eliminating the IEF in GaN-based materials is highly expected to improve the efficiency of lighting devices. Many efforts have been devoted to this. Doping, which may be the earliest attempt to screen the electrostatic field, fails, because the doping concentrations required to achieve the magnitude of the field are as high as $10^{19} \mathrm{~cm}^{-319,20}$. A promising way to overcome the fundamental challenges is to realize semi- or nonpolar materials that can sharply reduce or even eliminate the IEF. In 2000, Ploog and colleagues $^{21}$ reported the molecular beam epitaxy (MBE) deposition technique by using $\mathrm{LiAlO}_{2}$ as a substrate to obtain nonpolar $\mathrm{GaN}$. Atomically flat surfaces have been achieved by the classic two-step approach or a high-temperature AIN buffer method ${ }^{22}$. However, the high density of stacking faults makes the quality of the materials far from the requirements for device structures. Another intuitive alternative is to replace the wurtzite phase by the unpolarized zinc-blende phase of nitride semiconductors. However, the intrinsic thermodynamic metastability and the lack of ideal substrates hinder the realization of device-quality material even after extensive efforts ${ }^{23}$. The fundamental solution to this problem is to find a stable alternative material without IEF but that maintains optoelectronic performance comparable to that of wurtzite structures.

Recently, diverse two-dimensional (2D) GaN-based semiconductors were explored by both theoretical and experimental scientists ${ }^{24-37}$. Interestingly, many reported structures (including hexagonal ${ }^{24-26}$, haeckelite $(4 \mid 8)^{27,28}$, and tetragonal ${ }^{29,34}$ ) were nonpolarized. Tsipas et al. ${ }^{32}$ obtained hexagonal AIN nanosheets by $\mathrm{MBE}$ on $\mathrm{Ag}(111)$ and confirmed the reduced band gap for hexagonal AIN in 2013. One year later, Malin et al. ${ }^{33}$ realized hexagonal AIN on the semiconductor substrate Si(111). However, the huge cost and uncontrollable quality of the sample obtained by MBE remarkably hindered the large-scale commercial application of 2D III-nitride materials. In the past 3 years, scientists from the Pennsylvania State University ${ }^{35}$ and South China University of Technology ${ }^{36,37}$ successfully synthesized 2D GaN and AIN by metal organic chemical vapor deposition with the assistance of graphene encapsulation. The exciting achievements significantly accelerated the steps of industrialization for 2D GaN-based semiconductors. Kolobov et al. $^{28}$ predicted that graphitic and wurtzite few-layer GaN was unstable and spontaneously reconstructed into the haeckelite structure, in which the interlayer bonds along the $c$-axis exhibited a $4 \mid 8$ motif (highlighted by yellow shadow), as shown in Fig. 1a. Notably, the [0001] and [000-1] surfaces with opposite charges in the wurtzite

\footnotetext{
'State Key Laboratory of Luminescence and Applications, Changchun Institute of Optics, Fine Mechanics and Physics, Chinese Academy of Sciences, Changchun 130033, China. ${ }^{2}$ Center of Materials Science and Optoelectronics Engineering, University of Chinese Academy of Sciences, Beijing 100049, China. ${ }^{凶}$ email: shizm@ciomp.ac.cn; lidb@ciomp.ac.cn
} 
(a)

(b)
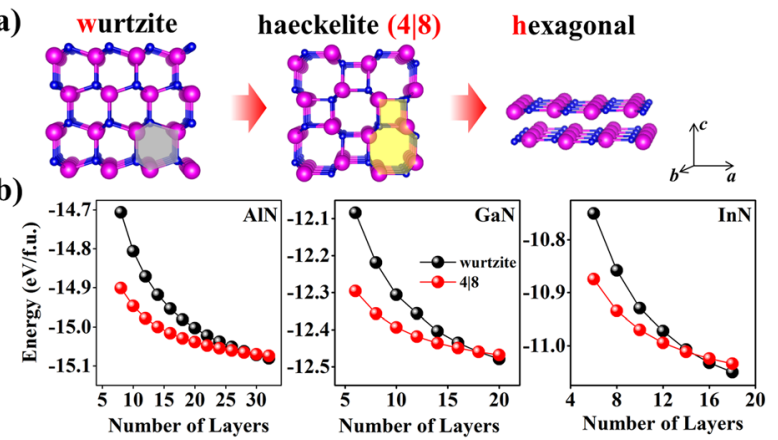

(c)

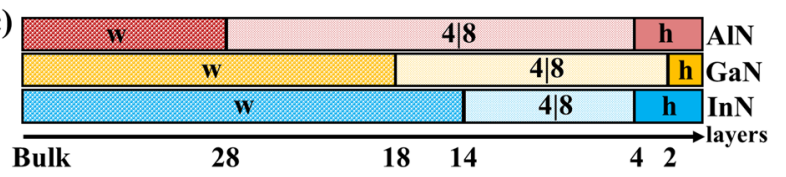

Fig. 1 Atomic structures and phase transition diagram for 2D GaN-based semiconductors. a Structural prototypes of wurtzite, 4| 8 , and hexagonal structures. The hexagonal ring in the wurtzite structure and the $4 \mid 8$ ring in the haeckelite structure are marked by gray and yellow shadows. b Free energy per formula unit as a function of the number of layers for wurtzite (black) and 4|8 (red) AIN, GaN, and InN slabs. c Scheme of the phase evolution with the number of layers.

configuration are replaced by neutral surfaces in $4 \mid 8$ configurations. The IEF should be reduced or even eliminated in these configurations. This is highly expected to increase the quantum efficiency of lighting devices based on GaN-based semiconductors.

First-principles calculations have obtained great success in the research of GaN-based semiconductors ${ }^{24,28,29,38,39}$. In this work, we theoretically demonstrated by first-principles calculations that in GaN-based semiconductors, the $4 \mid 8$ configuration is more energetically favorable than the wurtzite configuration after reducing the dimensions from bulk to $2 \mathrm{D}$ structures. This supports a convenient method to obtain GaN-based semiconductor (represented by AIN, GaN, and InN in this work) samples without IEF. The structural phase transition of wurtzite $(w) \rightarrow$ haeckelite (4 | 8 ) $\rightarrow$ hexagonal (h) induced by surface energy is revealed, as shown in Fig. 1a. Compared with the wurtzite configuration, the 4|8 configuration possesses comparable optoelectronic performance but no IEF. Meanwhile, we also provide a feasible approach, "thickness-controlled" vdW epitaxy, to promote the experimental realization of the $4 \mid 8$ configuration. We find that the surface atomic structure of the substrate and the substrate/ epilayer interaction are two decisive factors determining the configuration of materials.

\section{RESULTS AND DISCUSSIONS}

\section{Structural stability}

In the bulk phase, the total free energies of the wurtzite configurations are smaller than those of the $4 \mid 8$ configurations by 78,110 , and $48 \mathrm{meV}$ for AIN, GaN, and InN, respectively, as the bond lengths and angles of the wurtzite configurations are closer to perfect tetrahedral coordination. The bond angles are all $\sim 109.5 \pm 1^{\circ}$ in the wurtzite configurations, which are close to those of the ideal tetrahedral configuration. Meanwhile, one bond angle decreases to $90 \pm 2^{\circ}$ and the others increase to $112.5 \pm 1^{\circ}$ in the $4 \mid$ 8 configurations, which significantly deviates from the ideal tetrahedral configuration. Therefore, the $4 \mid 8$ configurations endure serious distortions and the distortion will accumulate with increasing thickness. The lattice parameters of the wurtzite and 4 | 8 configurations are listed in Table 1. For 2D structures, the stability of the materials is contributed by both the bulk and
Table 1. Lattice parameters and band gaps for different phases of group-III nitrides.

\begin{tabular}{llllll|}
\hline Structures & \multicolumn{2}{l}{$\begin{array}{l}\text { Lattice } \\
\text { parameters }(\AA)\end{array}$} & & \multicolumn{2}{l}{ Band gap $(\mathrm{eV})$} \\
\cline { 2 - 3 } \cline { 5 - 6 } & $a$ & $c$ & & PBE & HSE06 \\
\hline w-AIN & 3.11 & 4.99 & & 4.17 & 5.98 \\
w-GaN & 3.19 & 5.21 & & 1.86 & 3.24 \\
w-InN & 3.54 & 5.69 & & Semi-metal & 0.69 \\
4|8-AIN & 3.11 & 5.30 & & 3.85 & 5.57 \\
4|8-GaN & 3.20 & 5.50 & & 1.86 & 3.24 \\
4|8-InN & 3.56 & 6.02 & & Semi-metal & 0.76 \\
h-AIN & 3.12 & & & 2.91 & 4.32 \\
h-GaN & 3.20 & & & 2.20 & 3.53 \\
h-InN & 3.57 & & 0.63 & 1.72 \\
\hline
\end{tabular}

surface parts. The calculated surface energies (defined in the methodology section) are as follows: $\gamma_{\text {AlN }}^{w}$ is $2.02 \mathrm{eV} \mathrm{f.u}^{-1}, \gamma_{\mathrm{GaN}}^{w}$ is $1.72 \mathrm{eV} \mathrm{f.u.}^{-1}, \gamma_{\mathrm{InN}}^{w}$ is $0.91 \mathrm{eV} \mathrm{f.u.}{ }^{-1}, \gamma_{\text {AIN }}^{4 / 8}$ is $0.93 \mathrm{eV} \mathrm{f.u} .^{-1}, \gamma_{\text {GaN }}^{4 / 8}$ is $0.74 \mathrm{eV}_{\text {f.u. }}{ }^{-1}$, and $\gamma_{\operatorname{lnN}}^{4 / 8}$ is $0.46 \mathrm{eV} \mathrm{f.u.}{ }^{-1}$. The $\gamma_{m}^{4 / 8}$ values are generally much lower than the $\gamma_{m}^{w}$ values regardless of the thickness (as shown in Supplementary Fig. 2), indicating that the surfaces of the $4 \mid 8$ configurations are obviously more stable than those of the wurtzite configurations. The surfaces of the wurtzite configurations were classified as type III by Tasker and are intrinsically unstable due to the divergence of the surface energy $\left(\gamma_{m}^{c}\right)$, as well as numerous dangling bonds ${ }^{40}$. The strong surface dipoles associated with the presence of oppositely charged cations or anions on each surface introduce instability into the wurtzite structures. These issues are considerably optimized in the $4 \mid 8$ structures. As shown in Fig. $2 \mathrm{~b}$, the outermost atomic planes in $4 \mid 8$ slabs are nearly flat, thus minimizing the surface dipoles and neutralizing the surface charge. However, with increasing thickness, the rise in energy induced by accumulated distortion becomes greater than the energy compensation of the surface energy, leading to the wurtzite configurations being more stable than the 4|8 configurations in thick films or bulk phases. According to our results, the $4 \mid 8$ slabs show lower energy than wurtzite slabs when $4<n \leq 28$ ( $n$ is the number of layers) for AIN, $2<n \leq 18$ for GaN, and $4<n \leq 14$ for $\operatorname{InN}$, as shown in Fig. $1 b, c$, although the wurtzite structures are the most stable configurations when $n>28$ for AIN, $n>18$ for GaN, and $n>14$ for $\operatorname{lnN}$. The wurtzite $\rightarrow 4 \mid 8$ transition occurs when the thickness is reduced to several nanometers. In fact, the dangling bonds on the surface are quite unstable; thus, we consider the hydrogen-passivated surface structures following the same method and similar trends are observed as shown in Supplementary Fig. 3.

As the thickness is continuously reduced to a few atomic layers, the $4 \mid 8$ configurations transform to hexagonal configurations. First, we extract buckled monolayers from bulk wurtzite or 4 | 8 structures as initial structures. After relaxation, we obtain planar hexagonal structures such as graphene or h-BN, as shown in Fig. 1a. To remove the dangling bonds and stabilize the surface, the tetrahedral coordination is reconstructed into triangle-planar coordination. However, the $s p^{2}$ hybridization is still not stable enough. If two monolayers are stacked together to form a bilayer, the $s p^{2}$ hybridization is slightly distorted and the interlayer distances are $2.14,2.65$, and $2.47 \AA$, which are much shorter than that in graphene $(3.30 \AA)$. Hence, the interlayer coupling is much stronger than the normal vdW interaction. The calculated $\gamma_{\text {AIN }}^{h}$ is $1.04 \mathrm{eV} \mathrm{f.u}^{-1}, \gamma_{\mathrm{GaN}}^{h}$ is $0.48 \mathrm{eV} \mathrm{f.u}{ }^{-1}$, and $\gamma_{\mathrm{InN}}^{h}$ is $0.86 \mathrm{eV} \mathrm{f.u}{ }^{-1}$, indicating the quasi-bond character between the layers of hexagonal structures. According to our calculations, the planar 

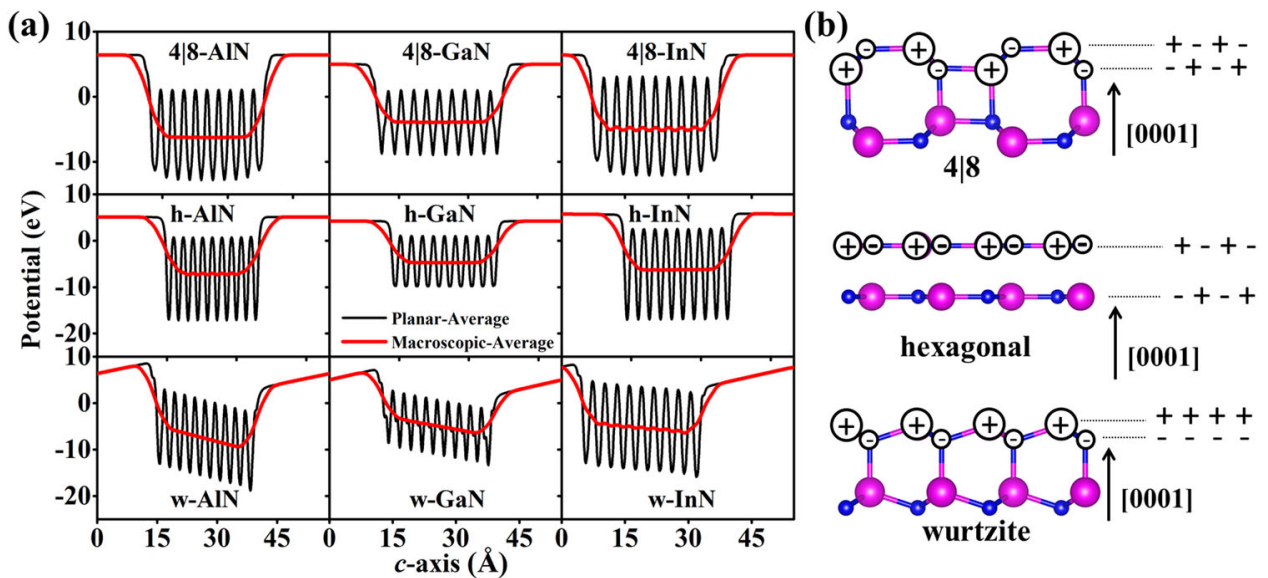

Fig. 2 Internal electrostatic potentials and the charge distributions for 2D GaN-based semiconductors in different configurations. a Planar-average (black line) and macroscopic-average (red line) internal electrostatic potentials along the $c$-axis for $4 \mid 8$, hexagonal, and wurtzite ten-layer slabs. The dangling bonds in $4 \mid 8$ and wurtzite on the surfaces are passivated by hydrogen atoms. $\mathbf{b}$ Scheme of charge distribution along the $c$-axis for $4 \mid 8$, hexagonal, and wurtzite configurations.

hexagonal structure can only be stable for at most two layers of $\mathrm{GaN}$ and $\operatorname{lnN}$, and four layers of AIN.

\section{Optoelectronic properties}

First, we employ the ten-layer slabs of 2D GaN-based semiconductors to evaluate the IEF of the $4 \mid 8$, hexagonal, and wurtzite configurations, as shown in Fig. 2a. We find that the IEF pointing from the metal toward the $\mathrm{N}$ surface is enhanced by decreasing the radius of the metal atoms. The magnitudes are calculated to be 22,16 , and $7 \mathrm{MV} \mathrm{cm}^{-1}$ for ten-layer w-AIN, w-GaN, and w-InN, respectively, as determined by the slope of the internal macroscopic-average electrostatic potential. We also repeat the results of the bilayer $\mathrm{w}-\mathrm{GaN}$ reported in ref. ${ }^{39}$ to confirm the reliability of the method. In contrast, the IEFs of the $4 \mid 8$ and hexagonal configurations are almost zero, demonstrating that the IEF is almost eliminated in 2D structures. This can be explained by the different charge distributions along the $c$-axis displayed in Fig. $2 \mathrm{~b}$. The $4 \mid 8$ and hexagonal configurations are formed by stacked neutral atomic layers, so the electrostatic potentials are equal for each layer, whereas positive (metal atoms) and negative ( $\mathrm{N}$ atoms) charged atomic layers are alternately stacked in the wurtzite configuration. This causes an electrostatic potential difference, which accumulates with increasing layer number. The results for the ten-layer slabs without hydrogen passivation are displayed in Supplementary Fig. 4. The same trend can be seen and the values are 16,10 , and $6 \mathrm{MV} \mathrm{cm}^{-1}$ for the wurtzite structures.

The ideal materials should not only be IEF free but also maintain optoelectronic properties comparable to those of the wurtzite configurations. The HSE06-level electronic band structures for the different configurations are displayed in Fig. 3 and the band gaps are listed in Table 1. First, the band structures of the bulk wurtzite structures are calculated as references and the band gaps are 5.98, 3.24, and $0.69 \mathrm{eV}$ for $\mathrm{w}-\mathrm{AIN}, \mathrm{w}-\mathrm{GaN}$, and $\mathrm{w}-\mathrm{InN}$, respectively. The calculated results agree with the experimental observations. The band structures of the bulk $4 \mid 8$ structures are almost the same as those for the bulk wurtzite structures and the band gaps are 5.57, 3.24 , and $0.76 \mathrm{eV}$, because the contribution of the band edge states has not been affected by the phase transition. The spatial wavefunction distributions of the valence band maximum (VBM) and conduction band minimum (CBM) are displayed in Fig. 4. We find that the CBM is always contributed by the $s$ orbital of $\mathrm{N}$ atoms for both the wurtzite and 4|8 structures; however, the contributions to the VBM are slightly different. Overall, the VBM is contributed by the $p$ orbital of the $\mathrm{N}$ atoms, but the component changes with the wurtzite-to-4|8 transition. The VBM is

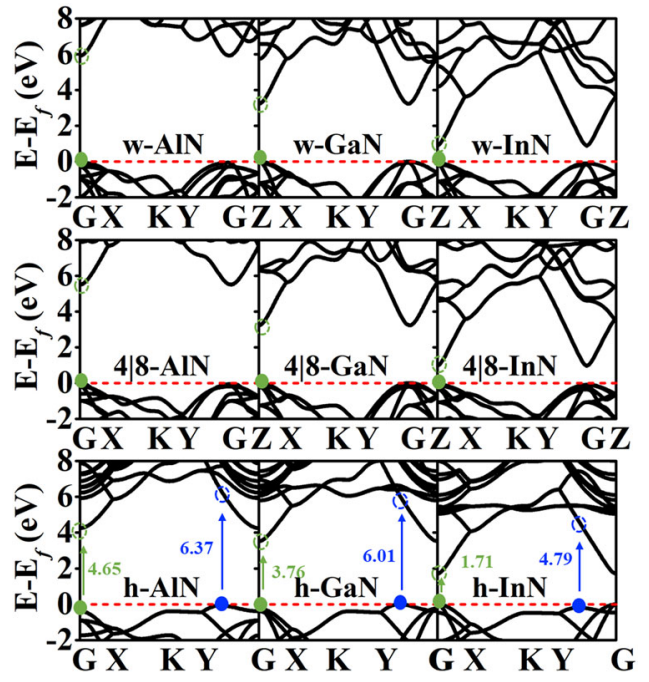

Fig. 3 Calculated band structures of w-, 4|8-, and monolayer group-III nitrides at the HSE06 level. The high symmetry k-points are $\mathrm{G}(0,0,0), \mathrm{X}(0.5,0,0), \mathrm{K}(0.5,0.5,0), \mathrm{Y}(0,0.5,0)$, and $\mathrm{Z}(0,0,0.5)$ in the cuboid Brillouin zone. The VBM and CBM at the G points for the wurtzite and $4 \mid 8$ band structures are marked by green solid and hollow circles, respectively. The arrows are the scheme of vertical excitation at the $G$ and $Q$ (between $G$ and $Y$ ) points for the hexagonal configurations. The blue color represents the vertical excitation at the $\mathrm{Q}$ point.

contributed by the $p_{\mathrm{z}}$ orbital for w-AIN, by the $p_{\mathrm{x}}$ orbital for 4 8 -AIN, by the $p_{\mathrm{x}}$ orbital for $\mathrm{w}-\mathrm{GaN}$, by the $p_{\mathrm{z}}$ orbital for $4 \mid 8-\mathrm{GaN}$, by the $p_{\mathrm{x}}$ orbital for $\mathrm{w}-\operatorname{InN}$, and by the $p_{\mathrm{y}}$ orbital for $4 \mid 8-\operatorname{InN}$. In the hexagonal structures, the VBM is between the $Y$ and $G$ points (defined as the $\mathrm{Q}$ point and marked by blue dots) and contributed by the $p_{z}$ orbital of $\mathrm{N}$ atoms, and the CBM is contributed by the hybridized state including $\mathrm{N}-\mathrm{s}$ and $\mathrm{Al} / \mathrm{Ga}-\mathrm{sp}$ for $\mathrm{AIN}$ and GaN; thus, the hexagonal structures show indirect band gaps. $\mathrm{h}-\ln \mathrm{n}$ is still a direct band gap material, because the VBM and CBM are contributed by the bonding and antibonding states of the N-s and In-sp orbitals. The band gaps are 4.32, 3.53, and $1.72 \mathrm{eV}$ for $\mathrm{h}$ AIN, h-GaN, and h-InN, respectively. Therefore, by reducing the thickness, the direct $\rightarrow$ indirect band gap transition occurs for AIN and GaN but not for InN. The Perdew-Burke-Ernzerhof parametrization (PBE) level band structure evolutions for wurtzite and 4 | 8 


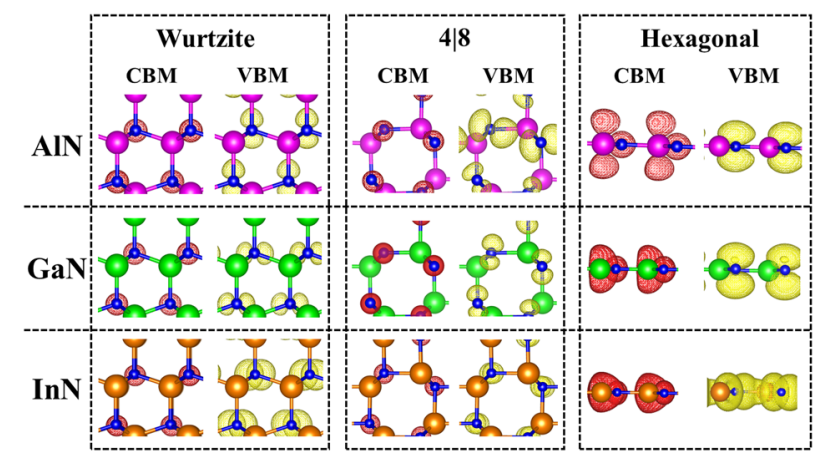

Fig. 4 Spatial band edge state distribution for different configurations of group-III nitrides. The isosurface is set to $6 \times 10^{-4} \mathrm{e}$ bohr $^{-3}$. Red and yellow colors represent the CBM and VBM, respectively. The purple, green, orange, blue, and white balls represent $\mathrm{Al}, \mathrm{Ga}$, In, N, and $\mathrm{H}$ atoms, respectively.

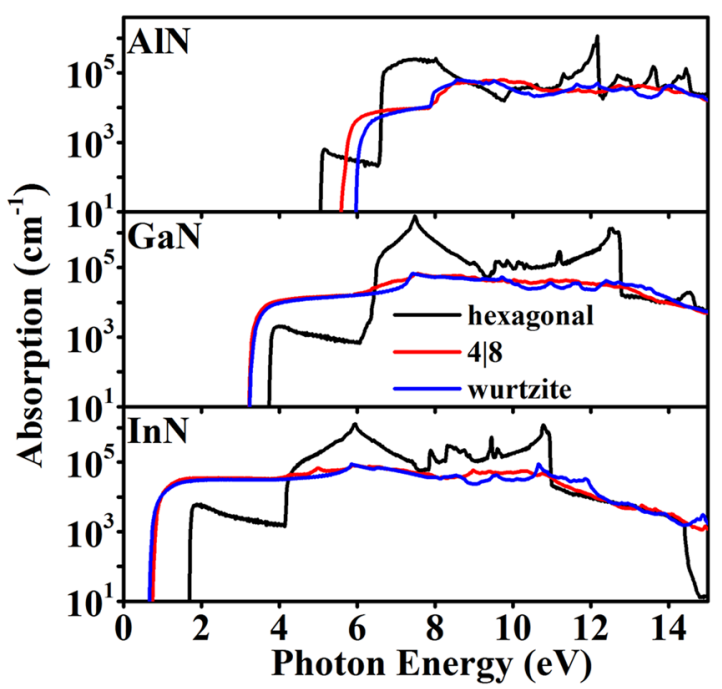

Fig. 5 Calculated absorption coefficients for different configurations of group-III nitrides. The results are obtained under the HSE06 level with the RPA.

2D III-nitrides with increasing thickness are exhibited in Supplementary Figs. 5-7.

The absorption coefficient and carrier effective mass for the hexagonal, $4 \mid 8$, and wurtzite structures are calculated and displayed in Fig. 5 and Table 2, which are key parameters for optoelectronic devices. First, the wurtzite (blue line) and $4 \mid 8$ (red line) configurations exhibit stronger band edge absorption intensities than the hexagonal (black line) configuration, as shown in Fig. 5; hence, the wurtzite and $4 \mid 8$ configurations are more suitable for application in lighting devices than the hexagonal configuration. Second, for the hexagonal configuration, the edge absorption intensities are at least one order of magnitude weaker than the second absorption. By analyzing the band structures, as shown in the insets of Fig. 3 (bottom row), we find that the band gaps at the $\mathrm{G}$ point (green) are $4.65,3.76$, and $1.71 \mathrm{eV}$, which match the band edge absorption. On the other hand, the band gaps at the $\mathrm{Q}$ point (blue) are 6.37, 6.01, and $4.79 \mathrm{eV}$, which agree with the second absorption peak in Fig. 5. The VBMs of h-AIN and $\mathrm{h}-\mathrm{GaN}$ are located at the $\mathrm{Q}$ points, so the absorption intensities are stronger than those at the $\mathrm{G}$ point. The electron effective mass is hardly changed for the different configurations based on a comparison of the results with those of wurtzite structures. We can find that the in-plane hole effective masses for the $4 \mid 8$ and hexagonal configurations are much smaller than those for the
Table 2. Carrier effective mass for different phases of group-III nitrides.

\begin{tabular}{|c|c|c|c|c|c|c|}
\hline \multirow[t]{2}{*}{ Structures } & \multicolumn{3}{|c|}{ Electron $\left(m_{0}\right)$} & \multicolumn{3}{|c|}{ Hole $\left(m_{0}\right)$} \\
\hline & $x$ & $y$ & $z$ & $x$ & $y$ & $z$ \\
\hline w-AIN & 0.31 & 0.31 & 0.29 & 4.24 & 4.28 & 0.25 \\
\hline w-GaN & 0.18 & 0.18 & 0.16 & 9.29 & 2.08 & 2.11 \\
\hline$w-\ln N$ & 0.05 & 0.05 & 0.05 & 2.19 & 2.19 & 2.14 \\
\hline $4 \mid 8-A I N$ & 0.33 & 0.35 & 0.33 & 0.64 & 2.58 & 3.27 \\
\hline $4 \mid 8-\mathrm{GaN}$ & 0.19 & 0.18 & 0.18 & 1.87 & 1.99 & 0.35 \\
\hline $4 \mid 8-\ln N$ & 0.03 & 0.03 & 0.05 & 0.09 & 0.07 & 2.12 \\
\hline h-AIN & 0.50 & 0.50 & & 1.28 & 1.18 & \\
\hline h-GaN & 0.24 & 0.24 & & 1.16 & 1.35 & \\
\hline$h-\ln N$ & 0.09 & 0.09 & & 1.30 & 1.53 & \\
\hline
\end{tabular}

wurtzite configurations. As the hole is the majority carrier in nitride materials, the in-plane mobility of the $4 \mid 8$ and hexagonal structures is much better than that of the wurtzite structures. This will improve the efficiency of horizontal structure optoelectronic devices.

\section{Substrates effect}

In experiments, wurtzite and hexagonal structures have already been realized, but the $4 \mid 8$ structure has not yet been observed, even though the $4 \mid 8$ structure is energetically favorable for nanothickness structures. This observation should be attributed to the substrate effect. Here we employ a four-layer $\mathrm{GaN}$ slab and two substrates, graphene (Gra) and silicon carbide ( $\mathrm{SiC}$ ), as examples to investigate the substrate effect. First, we cover the wurtzite or $4 \mid 8$ slabs on graphene, acting as vdW epitaxy ${ }^{41,42}$. After structural relaxation, the $4 \mid 8$ structure remains in the initial configuration, as shown in Fig. $6 a$, indicating the good stability of the $4 \mid 8$ structures for the four-layer GaN. However, the wurtzite configuration is too unstable to be maintained and changes into the $4 \mid 8$ configuration, as shown in Fig. 6 b. However, the situation for the SiC substrate is entirely different. Both the wurtzite and 4 | 8 configurations maintain their initial states after structural relaxation. The interaction between the four-layer GaN slab and $\mathrm{SiC}$ resulted in a different behavior. For freestanding four-layer $\mathrm{GaN}$, the 4|8 configuration is more stable than the wurtzite configuration. After stacking on graphene, the interaction between the four-layer $\mathrm{GaN}$ slab and graphene is weak and cannot restrict the phase transition. On the other hand, the fourlayer GaN slab bonded on the $\mathrm{SiC}$ substrate has a much stronger interaction than that on graphene, so the interaction is hard to overcome. It is observed from Fig. 6 (middle row) that charge transfer is almost negligible at the graphene/GaN interface but is quite obvious at the $\mathrm{SiC} / \mathrm{GaN}$ interface. Moreover, the structure of the SiC surface matches that of $w-G a N$, so $w-G a N$ is more energetically favorable than $4 \mid 8-\mathrm{GaN}$ on the SiC substrate by $63.9 \mathrm{meV}^{-2}$.

To consider the temperature effect, ab initio molecular dynamics (AIMD) is employed. The temperature is set to $1000 \mathrm{~K}$, which refers to the experimental environment ${ }^{35}$. The total energy of the system converged after a 4 ps AIMD simulation, as shown in Supplementary Fig. 8, and the structures at the end of the simulations are displayed in the bottom row of Fig. 6. The graphene and materials are separated due to the destruction of the weak interaction. The atoms of $4 \mid 8-\mathrm{GaN}$ just vibrate around the equilibrium position and the w-GaN transformed to $4 \mid 8-\mathrm{GaN}$, indicating that $1000 \mathrm{~K}$ is high enough to overcome the wurtziteto-4 $\mid 8$ phase transition barriers $\left(0.30,0.31\right.$, and $0.28 \mathrm{eV} \mathrm{f.u.}^{-1}$ for AIN, GaN, and InN, respectively, as shown in Supplementary Fig. 9). 


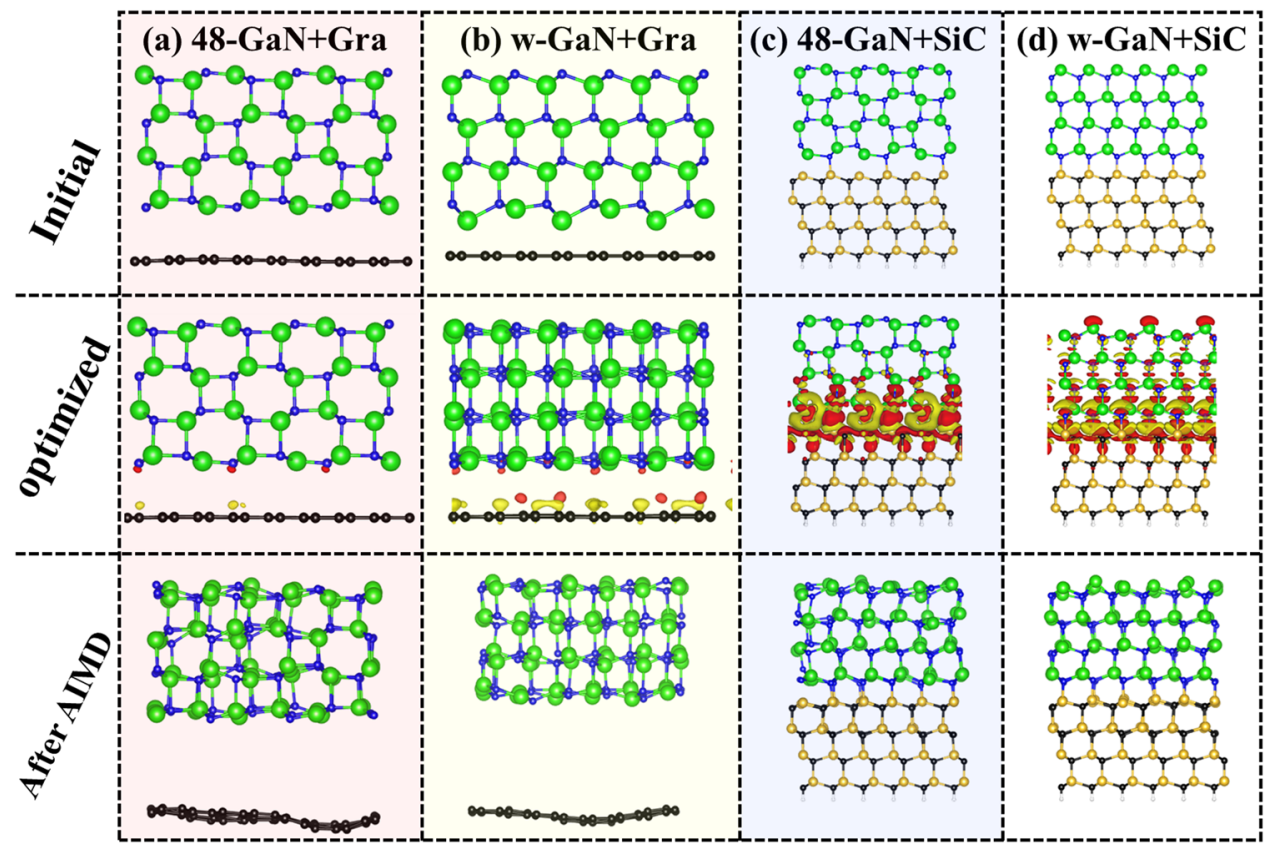

Fig. 6 Structures of four-layer wurtzite and 4 | 8-GaN on a graphene or SiC substrate. a 4 | 8-GaN on graphene, b w-GaN on graphene, c 4 | $8-\mathrm{GaN}$ on $\mathrm{SiC}$, and $\mathbf{d} \mathrm{w}-\mathrm{GaN}$ on $\mathrm{SiC}$. The top row shows the initial structures; the middle row shows the optimized structures with charge difference contours, where the isosurface is set to $1.5 \times 10^{-3} \mathrm{ebohr}^{-3}$; and the bottom row shows the snapshots after AIMD simulations at $1000 \mathrm{~K}$.

On the SiC substrate, w-GaN is still stable and $4 \mid 8-\mathrm{GaN}$ on $\mathrm{SiC}$ transforms to the wurtzite configuration, demonstrating that w$\mathrm{GaN}$ is more stable than $4 \mid 8-\mathrm{GaN}$. Therefore, $4 \mid 8$ configuration materials are expected to be experimentally achieved if we use a weakly bonded substrate such as graphene and control the thickness of the materials to within several nanometers by controlling the growth time. As far as we know, the reported experimental work for the growth of $2 \mathrm{D} \mathrm{GaN}$ and AIN used SiC or $\mathrm{Si}$ as substrates ${ }^{35-37}$. The interface interaction is strong and the surface structures match $w-G a N$. The growth temperature is usually as high as $1000 \mathrm{~K}$; thus, the $4 \mid 8$ configuration prefers to transform to wurtzite under this condition.

In summary, we have theoretically provided an effective approach, reducing the thickness, to overcome the problem of strong IEF in wurtzite GaN-based semiconductors. The 4/8 configuration is predicted to be the most stable configuration when the thickness is reduced to several nanometers. We reveal the wurtzite $\rightarrow 4 \mid 8 \rightarrow$ hexagonal phase transition as the thickness decreases. Interestingly, the $4 \mid 8$ configurations exhibit almost zero IEF, whereas the optoelectronic properties are comparable to those of the wurtzite configurations. The in-plane hole mobility for the hexagonal and $4 \mid 8$ structures is much better than that for the wurtzite structures. The substrate plays an important role in determining the configurations of the materials. The $4 \mid 8$ configuration is expected to be achieved by using the so-called "thickness-controlled" vdW substrates such as graphene and the wurtzite configuration is energetically favorable on $\mathrm{SiC}$ or $\mathrm{Si}$ substrates. Our results demonstrate that 2D GaN-based semiconductors exhibit many desirable properties and show great potential for application in novel optoelectronic devices.

\section{METHODS}

\section{General calculation details}

First-principles calculations were performed by employing the generalized gradient approximation (PBE) and projector-augmented wave method, as implemented in the Vienna Ab initio Simulation Package (VASP) ${ }^{43}$. A dispersion correction (DFT-D3) was used to incorporate the long-range $v d W$ interaction ${ }^{44,45}$. The unit cell for the $4 \mid 8$ structure was rectangular and contained eight atoms. To be comparable, we used the same supercell for the hexagonal and wurtzite structures in our calculations. The lattice stability of the bulk $4 \mid 8$ configurations for group-III nitrides was confirmed by phonon spectrum calculations, as displayed in Supplementary Fig. 1. The simulation cells were built with vacuum slabs thicker than $20 \AA$ to avoid artificial interaction of periodic images. The plane-wave basis set with a kinetic energy of $500 \mathrm{eV}$ was used. We employed $5 \times 8 \times 1$ Monkhorst-Pack $k$-grids in self-consistent calculations and $51 k$-points between high symmetry points of the Brillouin zone in the band structure calculations. In addition, the force and total energy convergence thresholds were set to $0.01 \mathrm{eV} \AA^{-1}$ and $10^{-6} \mathrm{eV}$, respectively. The lattice parameter differences were $<1 \%$ compared with the experimental results of wurtzite structures, as shown in Table 1.

HSE band structures and absorptions

To avoid the underestimation of the band gap from the PBE function, the hybrid exchange and correlation functional of Heyd-Scuseria-Ernzerhof $(\mathrm{HSE06})^{46}$ was used to obtain an accurate band structure. Here we used a mixing parameter $(a)$ of $33 \%$ for AIN and $31 \%$ for GaN and $\operatorname{lnN}$ so that band gaps of 5.98, 3.24, and $0.69 \mathrm{eV}$, respectively, were obtained for the wurtzite structures. Absorption spectra were calculated based on HSE06 including the random phase approximation. The HSEO6 and absorption spectra were obtained by the PWmat code ${ }^{47}$.

Nudged elastic band and phonon

The climbing image nudged elastic band method ${ }^{48,49}$ using eight images to connect the initial and final configurations (force convergence of $0.01 \mathrm{eV} \AA^{-1}$ ) was employed to obtain the structural phase transition barrier. Force constants for phonon calculations were found by using density functional perturbation theory ${ }^{50}$, as implemented in VASP. On the basis of the calculated force constants, phonon dispersion curves were obtained with the PHONOPY package ${ }^{51}$.

Ab initio molecular dynamics

Finally, $3 \times 3$ supercells (more than 300 atoms) were used for AIMD simulations with the canonical ensemble, which were performed to consider the temperature effect on the structural transition at $1000 \mathrm{~K}$ (Nosé-Hoover thermostat, total simulation time of $3 \mathrm{ps}$, time step of $1 \mathrm{fs}$ ). AIMD was carried out using the CP2K package ${ }^{52}$ with the hybrid Gaussian 
and plane wave method implemented in the Quickstep module ${ }^{53}$. Threedimensional periodic boundary conductions were applied for the system under NVT conditions. Temperature control was implemented for the ionic degrees of freedom by using Nosé-Hoover thermostats. The PBE exchange and correlation functional was used in combination with Godecker-Teter-Hutter (GTH $)^{54}$ pseudopotentials and the DZVPMOLOPT-SR-GTH basis sets ${ }^{55}$. The auxiliary plane-wave basis was set to an energy cutoff of 300 Ry and the time step was set to $1 \mathrm{fs}$.

\section{Surface energy}

The surface energy $\left(\gamma_{m}^{c}\right)$ for different materials $(m=\mathrm{AIN}, \mathrm{GaN}$, or $\operatorname{lnN})$ in different atomic configurations $(c=w, 4 \mid 8$, or $h$ ) per molecular formula unit (f.u.) was employed to estimate the stability of the 2D group-III nitride slabs, which is defined as:

$\gamma_{m}^{c}=\left(E_{\text {bulk }}-E_{\text {slab }}\right) / 2 N$

$E_{\text {slab }}$ and $E_{\text {bulk }}$ represent the total energy of slabs (contain two dangling surfaces) and bulk materials (the number of atoms is the same as in the slab), respectively. $N$ is the number of molecular formula units on the surface. A high $\gamma_{m}^{c}$ implied poor stability.

\section{Carrier effective mass}

The carrier effective mass $\left(m_{e / h}^{*}\right)$ obtained by fitting the band edge was calculated according to the following equation:

$m_{e / h}^{*}=\left[\hbar^{2} \frac{\partial^{2} \varepsilon(\mathbf{k})}{\partial \mathbf{k}^{2}}\right]^{-1}$

where $\hbar$ is the reduced $\partial$ Planck constant, $\varepsilon(k) \varepsilon(\mathbf{k})$ is the band edge eigenvalue, and $\mathbf{k}$ is the wavevector. A small $m_{e / h}^{*}$ implied good carrier mobility. In this work, we studied $m_{e / h}^{*}$ along $\Gamma(0,0,0) \rightarrow X(0.5,0,0), \Gamma \rightarrow Y$ $(0,0.5,0)$, and $\Gamma \rightarrow Z(0,0,0.5)$.

\section{DATA AVAILABILITY}

All data generated or analyzed during this study are included in this published article (and its Supplementary Information files), and are available from the authors.

\section{CODE AVAILABILITY}

The calculations were implemented using the VASP, PWmat, CP2K, and Phonopy packages.

Received: 2 June 2020; Accepted: 31 July 2020;

Published online: 28 August 2020

\section{REFERENCES}

1. Mohammad, S. N. \& Morkoç, H. Progress and prospects of group-III nitride semiconductors. Prog. Quant. Electron. 20, 361-525 (1996).

2. Nakamura, S., Mukai, T. \& Senoh, M. Candela-class high-brightness InGaN/AIGaN double-heterostructure blue-light-emitting diodes. Appl. Phys. Lett. 64, 1687-1689 (1994).

3. Nakamura, S. \& Krames, M. R. History of gallium-nitride-based light-emitting diodes for illumination. Proc. IEEE 101, 2211-2220 (2013).

4. Li, D. et al. Realization of a high-performance GaN UV detector by nanoplasmonic enhancement. Adv. Mater. 24, 845-849 (2012).

5. Sun, Y. et al. Room-temperature continuous-wave electrically pumped InGaN/ GaN quantum well blue laser diode directly grown on Si. Light Sci. Appl. 7, 1-7 (2018).

6. Lim, S.-H. et al. Electrically driven, phosphor-free, white light-emitting diodes using gallium nitride-based double concentric truncated pyramid structures. Light Sci. Appl. 5, e16030 (2016).

7. Morkoc, H. et al. Large-band-gap SiC, III-V nitride, and II-VI ZnSe-based semiconductor device technologies. J. Appl. Phys. 76, 1363-1398 (1994).

8. Li, D. et al. Influence of threading dislocations on GaN-based metalsemiconductor-metal ultraviolet photodetectors. Appl. Phys. Lett. 98, 011108 (2011).

9. Li, D., Jiang, K., Sun, X. \& Guo, C. AlGaN photonics: recent advances in materials and ultraviolet devices. Adv. Opt. Photonics 10,43-110 (2018).
10. Nakamura, S. et al. InGaN/GaN/AIGaN-based laser diodes with modulation-doped strained-layer superlattices grown on an epitaxially laterally overgrown GaN substrate. Appl. Phys. Lett. 72, 211-213 (1998).

11. Li, Z. et al. Suppression of thermal degradation of InGaN/GaN quantum wells in green laser diode structures during the epitaxial growth. Appl. Phys. Lett. 103, 152109 (2013)

12. Zhang, Y. et al. Effects of a step-graded $\mathrm{Al}_{\mathrm{x}} \mathrm{Ga}_{1-\mathrm{x}} \mathrm{N}$ electron blocking layer in InGaN-based laser diodes. J. Appl. Phys. 109, 083115 (2011).

13. Bernardini, F., Fiorentini, V. \& Vanderbilt, D. Spontaneous polarization and piezoelectric constants of III-V nitrides. Phys. Rev. B 56, R10024 (1997).

14. Fiorentini, V. et al. Effects of macroscopic polarization in III-V nitride multiple quantum wells. Phys. Rev. B 60, 8849 (1999).

15. Bernardini, F. \& Fiorentini, V. Macroscopic polarization and band offsets at nitride heterojunctions. Phys. Rev. B 57, R9427 (1998).

16. Langer, R. et al. Giant electric fields in unstrained GaN single quantum wells. Appl. Phys. Lett. 74, 3827-3829 (1999).

17. Morkoc, H. \& Mohammad, S. N. High-luminosity blue and blue-green gallium nitride light-emitting diodes. Science 267, 51-55 (1995).

18. Deguchi, T. et al. Quantum-confined stark effect in an AIGaN/GaN/AIGaN single quantum well structure. Jpn J. Appl. Phys. 38, L914 (1999).

19. Thamm, A. et al. Optical properties of heavily doped $\mathrm{GaN} /(\mathrm{Al}, \mathrm{Ga}) \mathrm{N}$ multiple quantum wells grown on $6 \mathrm{H}-\mathrm{SiC}(0001)$ by reactive molecular-beam epitaxy. Phys. Rev. B 61, 16025 (2000).

20. Jiang, $\mathrm{H}$. et al. Photoluminescence and photoluminescence excitation spectra of $\mathrm{In}_{0.2} \mathrm{Ga}_{0.8} \mathrm{~N}-\mathrm{GaN}$ quantum wells: comparison between experimental and theoretical studies. IEEE J. Quantum Electron. 35, 1483-1490 (1999).

21. Waltereit, P. et al. Nitride semiconductors free of electrostatic fields for efficient white light-emitting diodes. Nature 406, 865-868 (2000).

22. Ranalli, F. et al. Non-polar AIN and GaN/AIN on r-plane sapphire. Phys. Stat. Solid. C 6, S780-S783 (2009).

23. Gil, B. Group III Nitride Semiconductor Compounds: Physics and Applicationss (Clarendon Press, 1998).

24. Freeman, C. L., Claeyssens, F., Allan, N. L. \& Harding, J. H. Graphitic nanofilms as precursors to wurtzite films: theory. Phys. Rev. Lett. 96, 066102 (2006).

25. Prete, M. S., Pulci, O. \& Bechstedt, F. Strong in-and out-of-plane excitons in twodimensional InN nanosheets. Phys. Rev. B 98, 235431 (2018).

26. Pashartis, C. \& Rubel, O. Alloying strategy for two-dimensional GaN optical emitters. Phys. Rev. B 96, 155209 (2017).

27. Gürbüz, E., Cahangirov, S., Durgun, E. \& Ciraci, S. Single layers and multilayers of $\mathrm{GaN}$ and AIN in square-octagon structure: stability, electronic properties, and functionalization. Phys. Rev. B 96, 205427 (2017).

28. Kolobov, A. et al. Instability and spontaneous reconstruction of few-monolayer thick GaN graphitic structures. Nano Lett. 16, 4849-4856 (2016).

29. Singh, A. K., Zhuang, H. L. \& Hennig, R. G. Ab initio synthesis of single-layer III-V materials. Phys. Rev. B 89, 245431 (2014).

30. Zhuang, H. L., Singh, A. K. \& Hennig, R. G. Computational discovery of single-layer III-V materials. Phys. Rev. B 87, 165415 (2013).

31. Kecik, D. et al. Fundamentals, progress, and future directions of nitride-based semiconductors and their composites in two-dimensional limit: a first-principles perspective to recent synthesis. Appl. Phys. Rev. 5, 011105 (2018).

32. Tsipas, P. et al. Evidence for graphite-like hexagonal AIN nanosheets epitaxially grown on single crystal Ag (111). Appl. Phys. Lett. 103, 251605 (2013).

33. Malin, T., Mansurov, V., Galitsyn, Y. G. \& Zhuravlev, K. In 2014 Int. Conference on Manipulation, Manufacturing and Measurement on the Nanoscale (3M-NANO) 308-311 (IEEE, 2014).

34. Singh, A. K. \& Hennig, R. G. Computational synthesis of single-layer GaN on refractory materials. Appl. Phys. Lett. 105, 051604 (2014).

35. Al Balushi, Z. Y. et al. Two-dimensional gallium nitride realized via graphene encapsulation. Nat. Mater. 15, 1166 (2016).

36. Wang, W. et al. Lattice structure and bandgap control of 2D GaN grown on graphene/Si heterostructures. Small 15, 1802995 (2019).

37. Wang, W. et al. 2D AIN layers sandwiched between graphene and Si substrates. Adv. Mater. 31, 1803448 (2019).

38. Xiao, Y. et al. Anharmonic multi-phonon nonradiative transition: an ab initio calculation approach. Sci. China Phys. Mech. 63, 277312 (2020).

39. Sanders, N. et al. Electronic and optical properties of two-dimensional GaN from first-principles. Nano Lett. 17, 7345-7349 (2017).

40. Tasker, P. The stability of ionic crystal surfaces. J. Phys. C Solid State Phys. 12, 4977 (1979).

41. Kim, J. et al. Principle of direct van der Waals epitaxy of single-crystalline films on epitaxial graphene. Nat. Commun. 5, 4836 (2014).

42. Shi, Z. et al. Construction of van der Waals substrates for largely mismatched heteroepitaxy systems using first principles. Sci. China Phys. Mech. 62, 127311 (2019). 
43. Kresse, G. \& Joubert, D. From ultrasoft pseudopotentials to the projector augmented-wave method. Phys. Rev. B 59, 1758 (1999).

44. Grimme, S. Semiempirical GGA-type density functional constructed with a longrange dispersion correction. J. Comput. Chem. 27, 1787-1799 (2006).

45. Barone, V. et al. Role and effective treatment of dispersive forces in materials: Polyethylene and graphite crystals as test cases. J. Comput. Chem. 30, 934-939 (2009).

46. Heyd, J., Scuseria, G. E. \& Ernzerhof, M. Hybrid functionals based on a screened Coulomb potential. J. Chem. Phys. 118, 8207-8215 (2003).

47. Jia, W. et al. Fast plane wave density functional theory molecular dynamics calculations on multi-GPU machines. J. Comput. Phys. 251, 102-115 (2013).

48. Sheppard, D., Terrell, R. \& Henkelman, G. Optimization methods for finding minimum energy paths. J. Chem. Phys. 128, 134106 (2008),

49. Sheppard, D. et al. A generalized solid-state nudged elastic band method. J. Chem. Phys. 136, 074103 (2012)

50. Baroni, S., De Gironcoli, S., Dal Corso, A. \& Giannozzi, P. Phonons and related crystal properties from density-functional perturbation theory. Rev. Mod. Phys. 73, 515 (2001).

51. Chaput, L., Togo, A., Tanaka, I. \& Hug, G. Phonon-phonon interactions in transition metals. Phys. Rev. B 84, 094302 (2011).

52. Hutter, J., lannuzzi, M., Schiffmann, F. \& VandeVondele, J. cp2k: atomistic simulations of condensed matter systems. Wiley Interdiscip. Rev. Comput. Mol. Sci. 4, 15-25 (2014).

53. VandeVondele, J. et al. Quickstep: fast and accurate density functional calculations using a mixed Gaussian and plane waves approach. Comput. Phys. Commun. 167, 103-128 (2005)

54. Goedecker, S., Teter, M. \& Hutter, J. Separable dual-space Gaussian pseudopotentials. Phys. Rev. B 54, 1703 (1996).

55. VandeVondele, J. \& Hutter, J. Gaussian basis sets for accurate calculations on molecular systems in gas and condensed phases. J. Chem. Phys. 127, 114105 (2007).

\section{ACKNOWLEDGEMENTS}

The research reported in this publication was supported by the National Science Fund for Distinguished Young Scholars (61725403), the National Natural Science Foundation of China $(61804152,61874118,61834008$, and 61922078), the Special Fund for Research on National Major Research Instruments (61827813) Key Research Program of Frontier Sciences, CAS (Grant Number ZDBS-LY-JSC026), the Jilin Provincial Science and Technology Department (20180201026GX), and the Youth Innovation Promotion Association of CAS. It was also supported by the CAS Talents Program

\section{AUTHOR CONTRIBUTIONS}

D. L. and Z. S. conceived the idea and provided advice on this work. Y. J., Z. H., W. H. Z. Q. and Z. S. carried out the calculations. Y. J. and Z. S. wrote the manuscript. K. J., Y. C., S. Z. and X. S. provided very useful comments. All authors participated in the discussions on the results.

\section{COMPETING INTERESTS}

The authors declare no competing interests.

\section{ADDITIONAL INFORMATION}

Supplementary information is available for this paper at https://doi.org/10.1038/ s41699-020-00165-1.

Correspondence and requests for materials should be addressed to Z.S. or D.L.

Reprints and permission information is available at http://www.nature.com/ reprints

Publisher's note Springer Nature remains neutral with regard to jurisdictional claims in published maps and institutional affiliations. Attribution 4.0 International License, which permits use, sharing, adaptation, distribution and reproduction in any medium or format, as long as you give appropriate credit to the original author(s) and the source, provide a link to the Creative Commons license, and indicate if changes were made. The images or other third party material in this article are included in the article's Creative Commons license, unless indicated otherwise in a credit line to the material. If material is not included in the article's Creative Commons license and your intended use is not permitted by statutory regulation or exceeds the permitted use, you will need to obtain permission directly from the copyright holder. To view a copy of this license, visit http://creativecommons. org/licenses/by/4.0/.

(c) The Author(s) 2020 